\title{
Meningeal
}

\section{Hemangiopericytoma with Intracranial Metastases in an HIV-Positive Male: Case Report and Review of the Literature}

\author{
Zachary Beatty $^{\mathrm{a}}$ Thomas Bergman ${ }^{\mathrm{b}}$ \\ ${ }^{a}$ University of Minnesota Medical School and ${ }^{\mathrm{b}}$ Department of Neurosurgery, \\ Hennepin County Medical Center, Minneapolis, Minn., USA
}

\section{Key Words}

Meningeal hemangiopericytoma $\cdot$ Hemangiopericytoma $\cdot$ AIDS $\cdot$ HIV $\cdot$ Brain neoplasms

\begin{abstract}
Background: Meningeal hemangiopericytoma is a rare, aggressive CNS tumor that tends to invade locally, metastasize, and has a high rate of recurrence. HIV classically increases the risk of 3 AIDS-defining malignancies: Kaposi's sarcoma, non-Hodgkin's lymphoma and invasive cervical cancer. More recently, considerable interest has been paid to the link between HIV and a wider range of non-AIDS-defining cancers. An HIV-positive patient with meningeal hemangiopericytoma is described.
\end{abstract}

Case Description: A 36-year-old HIV-positive male presented with worsening headache and ataxia. The patient had experienced similar neurologic symptoms 4 months prior and MRI at that time had showed an extra-axial left cerebellar mass most consistent with benign meningioma. Repeat MRI showed the tumor had increased in size by a factor of greater than 20 in this 4-month period, with 4 small additional foci of similar enhancement. Subtotal resection was performed on the mass and final pathological diagnosis was meningeal hemangiopericytoma.

Conclusions: This represents the first reported case of meningeal hemangiopericytoma in an HIV-positive patient. This is also the shortest time to intracranial metastasis ever reported for a meningeal hemangiopericytoma. Although the increased risk in the HIV-positive population of non-AIDS-defining cancers that has been observed in recent years can largely be attributed to cancers with a known viral pathogenesis, it is speculated that HIV infection in this patient may have contributed to the occurrence or unique behavior of this rare tumor. 


\section{Introduction}

Meningeal hemangiopericytoma is a rare, aggressive CNS tumor accounting for $2.5 \%$ of all meningeal tumors and $1 \%$ of intracranial tumors as a whole [1]. It was initially considered to be a subset of meningioma but, in 1993, was reclassified as a separate entity by the World Health Organization [1]. Hemangiopericytoma can be clinically distinguished from meningioma based on its tendency to invade locally, metastasize, and recur $[2,3]$. Metastasis tends to be extraneural and recurrence rates can reach as high as $92 \%$ at 15 years [1]. To our knowledge, this is the first report of a meningeal hemangiopericytoma in an HIV-positive patient. Additionally, this represents the shortest time to intracranial metastasis ever reported in a meningeal hemangiopericytoma.

\section{Case Report}

\section{History and Diagnosis}

A 36-year-old HIV-positive male was admitted to our institution due to a 2-week history of worsening headache and ataxia. He was originally diagnosed with HIV 8 months prior and had begun antiretroviral therapy at that time, including a non-nucleoside reverse transcriptase inhibitor, efavirenz. Then, 4 months prior to this admission, the patient had experienced progressive ataxia and lower extremity paresthesias eventually leading to a brain MRI which showed an avidly enhancing extra-axial left cerebellar mass (fig. 1a) most consistent with benign meningioma. CD 4+ count at that time was 44. No mass effect was noted and watchful waiting was elected at that time. The patient's symptoms were stable until the clinical decline preceding this most recent admission. Repeat MRI at this time showed that the cerebellar mass had expanded over a period of 4 months from $1.6 \times 1.6 \times$ $1.5 \mathrm{~cm}$ to $4.8 \times 4.4 \times 4.0 \mathrm{~cm}$ with effacement of the 4 th ventricle, obstructive hydrocephalus, and additional small foci of similar enhancement located extra-axially at distant but still intracranial sites (fig. 1b, c). It was presumed that these additional foci represented spread of the primary process. At this point, the lesions were suspicious for atypical meningioma or hemangiopericytoma, primary CNS lymphoma, or infectious etiologies. Serum was negative for toxoplasma IgG. Cerebrospinal fluid was negative for Epstein-Barr virus and cytomegalovirus by polymerase chain reaction. Aerobic, anaerobic, fungal, viral, and acid-fast bacilli cultures of the cerebrospinal fluid were all negative.

\section{Operation}

The patient was brought to surgery to excise the tumor due to rapid enlargement and mass effect. Following posterior fossa craniotomy and microscope-aided cerebellar dissection, the tumor was identified as a rubbery yellow mass. Intra-operative frozen section specimens were labelled meningioma or hemangiopericytoma. Subtotal resection was then performed as the tumor was found to be extending through the tentorium. It was felt that adequate debulking had been performed despite leaving a small amount of residual tumor above the tentorium.

\section{Histopathological Findings and Postoperative Course}

Examination of the permanent sections showed a densely cellular neoplasm comprised of spindle cells arranged in a solid and storiform pattern. The background blood vessels had a distinct staghornlike pattern. Based on these features, the final diagnosis was hemangiopericytoma. The additional foci observed radiographically were not biopsied but were presumed to be metastases from the primary lesion. Following the surgery, the patient's neurologic symptoms were stable and the extraventricular drain was able to be removed on postoperative day 6 . On postoperative day 10 , the patient was discharged to a neurologic rehabilitation facility and after a short stay was discharged home. The patient underwent postoperative radiation therapy but, unfortunately, experienced a large recurrence of the cerebellar mass that required further surgery as well as interval increase in size in the accessory foci. The patient was undergoing additional rehabilitation at the time of submission of this work. 


\section{Discussion}

Meningeal hemangiopericytoma is a rare CNS tumor characterized by its local aggressiveness and tendency to recur and metastasize. In the largest published review of meningeal hemangiopericytoma, including follow-up data on 277 patients, local recurrence was seen in $57 \%$ of patients and metastasis was seen in $27 \%$ [3]. What makes the tumor seen in our patient unique is the speed and location of its metastases. Metastases of meningeal hemangiopericytoma typically take years to develop and spread to extra-neural sites in the vast majority of cases [2, 4]. In 2 large case series, the average time from initial excision to extraneural metastasis was 99 and 131 months, and only $1 / 10$ and $2 / 10$ of patients with metastasis, respectively, had spread to intracranial sites $[1,5]$. As for intracranial metastases specifically, they have been observed between 9 and 15 years after the initial excision and have either been concurrent with or following local recurrence in 3 cases for which these data are reported $[1,6]$. In our case, metastasis was intracranial and occurred within a period of 4 months, making this the shortest time to intracranial metastasis ever reported. Had initial cranial imaging been delayed by a matter of weeks, it is likely that this would have instead been a presentation of a multifocal primary meningeal hemangiopericytoma, which has never been reported in the literature [6]. Admittedly, without biopsy it is impossible to be certain that these additional small foci do in fact represent metastases, especially considering the numerous potential etiologies of CNS lesions seen with HIV. Although CNS lesions of multiple distinct etiologies in a single patient can occur with HIV, this is seen in a small proportion of patients $-6 \%$ in 1 case series of stereotactic brain biopsies in patients with HIV [7]. It is therefore reasonable to assume that these foci represent metastases of the primary lesion based on similar radiographic appearance.

Naturally, the question arises as to whether HIV infection was related to the genesis or uniquely aggressive behavior of this tumor. There is no known link between HIV infection and meningeal hemangiopericytoma, although there have been cases reported of non-meningeal hemangiopericytoma in HIV-positive patients [8, 9]. HIV infection is classically associated with an increased risk for three AIDS-defining malignancies, these being Kaposi's sarcoma, non-Hodgkin's lymphoma, and invasive cervical cancer [10]. These three malignancies have an identified viral pathogenesis and, as would be expected intuitively, the incidence and mortality of these malignancies in the HIVpositive population has decreased dramatically in the era of highly active antiretroviral therapy (HAART) $[11,12]$. Although HAART has pushed the available follow-up period for HIV-positive patients from years to decades, an increased incidence in many nonAIDS-defining cancers (NADCs) has been uncovered [11,12]. The standardized incidence ratio (SIR) for NADCs was observed to be as high as 2.49 in the HIV-positive population between 2002 and 2007 [12]. In addition to impaired defense against oncogenic viruses, this phenomenon has been proposed to involve impaired immune surveillance and even HAART itself (specifically non-nucleoside reverse transcriptase inhibitors, which the patient in our case was treated with) [11,12]. Meningeal hemangiopericytoma is so rare that a modest SIR such as 2.49 with HIV would not reveal itself with any statistical certainty without an incredibly large sample size. That being said, it is not unreasonable to entertain the possibility that HIV infection could be responsible for an increased risk or the accelerated growth of a rare tumor like 
meningeal hemangiopericytoma, but we simply lack the statistical power to show that link conclusively.

Of course it could be pure coincidence that this extraordinarily aggressive example of an already rare and aggressive tumor occurred in an HIV-positive patient. The elevated relative risk for NADCs that has been observed with HIV infection is due primarily to only a handful of cancers [12]. Two NADCs with the most consistently observed statistically significant risk increase with HIV infection are Hodgkin's lymphoma and anal cancer $[11,12]$. These two cancers have an identified viral pathogenesis, a trait they share with the AIDS-defining cancers. If cancers with a known viral pathogenesis are excluded, the increased risk of NADCs among those with HIV is much less striking. For example, the aforementioned SIR of 2.49 for all NADCs in the years 2002-2007 was calculated from an HIV-positive study cohort with 88 observed NADCs versus an expected incidence of 35.29 based on age- and sex-adjusted rates in the general population. Of these 88 NADCs, 46 were either Hodgkin's lymphoma or anal cancer [12]. Excluding observed and expected incidences of these two cancers, the SIR for NADCs drops from 2.49 to 1.22. Additionally, other cancers contributing heavily to the remaining increased observed rate of NADCs include lung cancer and cancers of the head and neck, cancers that are strongly linked to cigarette smoking. Smoking rates are exceptionally high among those with HIV, as high as 51\% [13], likely accounting for much of this remaining risk increase [11]. Thus impaired resistance to oncogenic viruses likely remains by far the most significant cause of increased cancer risk by HIV infection. Since there is no known link between meningeal hemangiopericytoma and any oncogenic virus, it seems less likely that HIV imparted any increased risk of occurrence for the tumor encountered in our case. Additionally, the aggressive behavior of this tumor seems likely to be coincidental as HIV-positive patients with more common primary intracranial NADCs such as astrocytoma appear to have outcomes similar to immunocompetent patients and tumor grade does not appear to be related to CD4+ count $[14,15]$.

While the weight of the data from larger cohorts suggests that the influence of HIV infection on the incidence or behavior of NADCs that lack a known viral pathogenesis is not clinically significant $[11,12]$, cases such as this one call this conclusion into question. To dismiss the extraordinary aggressiveness of this tumor as merely coincidental to HIV infection due to a lack of precedent seems irresponsible. Our understanding of the relationship between HIV and cancer is far from complete and will continue to evolve as follow-up of HIV-positive patients is increasingly measured in decades instead of years. This case is a perfect example of why this relationship deserves further inquiry. 

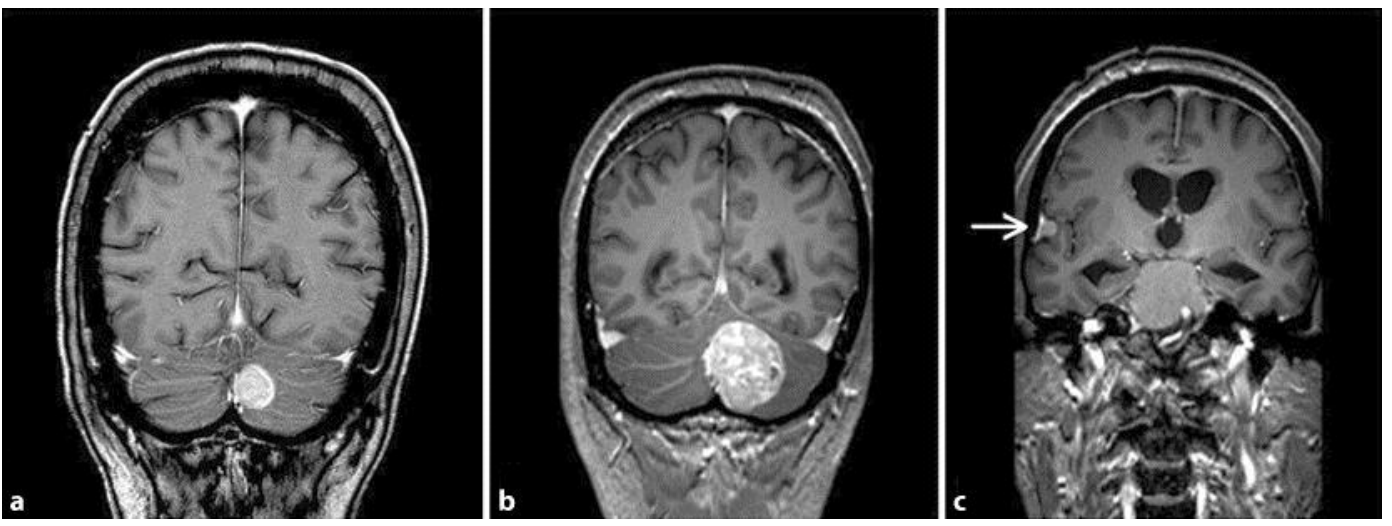

Fig. 1. Coronal $\mathrm{T}_{1}$-weighted contrast-enhanced MR images. a Image obtained 4 months prior to admission shows an avidly enhancing extra-axial left cerebellar mass. $\mathbf{b}$ Image obtained shortly after admission shows dramatic growth of the left cerebellar mass. c Same study as in $\mathbf{b}$; this image shows an additional small nodular extra-axial mass of similar contrast enhancement to the primary lesion (arrow).

\section{References}

1 Schiariti M, Goetz P, El-Maghraby H, Tailor J, Kitchen N: Hemangiopericytoma: long-term outcome revisited. Clinical article. J Neurosurg 2010;114:747-755.

- 2 Kim JH, Jung HW, Kim YS, Kim CJ, Hwang SK, Paek SH, Kim DG, Kwun BD: Meningeal hemangiopericytomas: long-term outcome and biological behavior. Surg Neurol 2003;59:47-54.

-3 Rutkowski MJ, Sughrue ME, Kane AJ, Aranda D, Mills SA, Barani IJ, Parsa AT: Predictors of mortality following treatment of intracranial hemangiopericytoma. J Neurosurg 2010;113:333-339.

-4 Borden NM: Aggressive angioblastic meningioma with multiple sites in the neural axis. AJNR Am J Neuroradiol 2009;16:793-794.

-5 Guthrie BL, Ebersold MJ, Scheithauer BW, Shaw EG: Meningeal hemangiopericytoma: histopathological features, treatment, and long-term follow-up of 44 cases. Neurosurgery 1989;25:514-522.

-6 Dufour H, Métellus P, Fuentes S, Murracciole X, Régis J, Figarella-Branger D, Grisoli F: Meningeal hemangiopericytoma: a retrospective study of 21 patients with special review of postoperative external radiotherapy. Neurosurgery 2001;48:756-763.

7 Gildenberg PL, Gathe JC Jr, Kim JH: Stereotactic biopsy of cerebral lesions in AIDS. Clin Infect Dis 2000;30:491-499.

8 Fleisher KE, Carlson ER, Schaberg SJ: Oropharyngeal mass. J Oral Maxillofac Surg 1998;56:359-364.

9 Lauinger MJ, Beadle N, Thisyakorn U: Ten-year-old boy with hemangiopericytoma and human immunodeficiency virus infection. Pediatr Infect Dis J 2001;20:321-323.

10 Centers for Disease Control: 1993 revised classification system for HIV infection and expanded surveillance case definition for AIDS among adolescents and adults. MMWR 1992;41:1-19.

-11 Engels EA, Biggar RJ, Hall HI, Cross H, Crutchfield A, Finch JL, Grigg R, Hylton T, Pawlish KS, McNeel TS, Goedert JJ: Cancer risk in people infected with human immunodeficiency virus in the United States. Int J Cancer 2008;123:187-194.

12 Powles T, Robinson D, Stebbing J, Shamash J, Nelson M, Gazzard B, Mandelia S, Møller H, Bower M Highly active antiretroviral therapy and the incidence of non-AIDS-defining cancers in people with HIV infection. J Clin Oncol 2009;27:884-890.

13 Collins RL, Kanouse DE, Gifford AL, Senterfitt JW, Schuster MA, McCaffrey DF, Shapiro MF, Wenger NS: Changes in health-promoting behavior following diagnosis with HIV: prevalence and correlates in a national probability sample. Health Psychol 2001;20:351-360.

14 Blumenthal DT, Raizer JJ, Rosenblum MK, Bilsky MH, Hariharan S, Abrey LE: Primary intracranial neoplasms in patients with HIV. Neurology 1999;52:1648-1651.

15 Bütnner A, Weis S: Non-lymphomatous brain tumors in HIV-1 infection: a review. J Neurooncol 1999;41:81-88. 